\title{
Treinamento em Esteira e Fortalecimento Muscular no Tratamento de Hemiparéticos Crônicos
}

\author{
Treadmill Training and Muscle Strengthening for Treat of Chronic Hemiparetic Stroke Patients
}

\begin{abstract}
Jonathan Borges de Castro ${ }^{1}$, Jenifer Cristina Abilel', Edna Maria Lavisió, Edna de Souza Cruz Uematsu', Juliano Viudes Moraes', Alexandre da Silva ${ }^{5}$
\end{abstract}

\begin{abstract}
RESUMO
Objetivo. analisar a aptidão cardiorrespiratória, o equilíbrio e a mobilidade de indivíduos hemiparéticos crônicos em resposta a um programa de treinamento em esteira e fortalecimento muscular. Método. Estudo quasi-experimental analítico, com participação de dez indivíduos hemiparéticos de ambos os sexos, com idade entre 40 e 74 anos que realizaram programa de treinamento com sessóes de 80 minutos, três vezes por semana durante quatro meses, avaliados por meio do Teste de Esforço em Esteira (TE), Performance-Oriented Mobility Assessment (POMA), Manovacuometria e mensuração da capacidade inspiratória máxima. $\mathrm{Na}$ análise estatística foram utilizados Teste $\mathrm{T}$ de Student e ANOVA com $\alpha=0,05$. Resultados. Houve aumento na média da distância percorrida de $145,4 \%$ no TE, este apresentou-se eficaz em $20 \%$ dos casos na avaliação inicial e em $70 \%$ dos casos na final. No teste POMA observou-se melhora de $24,7 \%$ no equilíbrio e $14,3 \%$ na marcha. A pressão inspiratória máxima aumentou 32,6\%, a expiratória $55,25 \%$ e a capacidade inspiratória $20 \%$. Conclusáo. Os resultados encontrados mostraram que o treinamento em esteira associado ao fortalecimento muscular melhorou a função cardiorrespiratória, a capacidade funcional, equilíbrio e mobilidade dos participantes deste estudo.
\end{abstract}

Unitermos. Acidente Cerebral Vascular, Reabilitação, Treinamento de Resistência, Exercício, Teste de Esforço.

Citaçáo. Castro JB, Abilel JC, Lavísio EM, Uematsu ESC, Moraes JV, Silva A. Treinamento em Esteira e Fortalecimento Muscular no Tratamento de Hemiparéticos Crônicos.

\begin{abstract}
Objective. To analyze the cardiorespiratory aptitude, the balance and mobility of chronic hemiparetic individuals in reply to a program of treadmill training and muscle strengthening. Method. Quasi-experimental analytic study, with the participation of 10 hemiparetic individuals of both sexes between 40 and 74 years old that had achievement program of training with sessions of 80 minutes, 3 days per week during 4 months, evaluated by means of the Stress Testing (ST), Performance-Oriented Mobility Assessment (POMA), Manovacuometry and measure of the maximum inspiratory capacity. We used paired Student T Test and two way ANOVA with $\alpha=0,05$. Results. The media of covered distance increased $145,4 \%$ in the ST; this one was effective in $20 \%$ of the cases in the initial evaluation and in $70 \%$ of the cases in the final evaluation. In POMA test were observed improvement of $24,7 \%$ in balance and $14,3 \%$ in march. The maximum inhaling pressure raised $32,6 \%$, the exhaling $55,25 \%$ and the inhaling capacity $20 \%$. Conclusion. The obtained results had shown that the treadmill training associated to muscle strengthening improved the cardiorespiratory function, the functional capacity, balance and mobility of the participants of this study.
\end{abstract}

Keywords. Stroke, Rehabilitation, Resistance Training, Exercise, Exercise Test.

Citation. Castro JB, Abilel JC, Lavísio EM, Uematsu ESC, Moraes JV, Silva A. Treadmill Training and Muscle Strengthening for Treat of Chronic Hemiparetic Stroke Patients.

\footnotetext{
Trabalho realizado na Universidade Cruzeiro do Sul, Sáo Paulo-SP, Brasil. 1. Fisioterapeuta, pós graduando em Fisioterapia Cardiorrespiratória e Hospitalar, UNICSUL, Sáo Paulo-SP, Brasil.

2. Médica Especialista em Cardiologia e UTI, Mestranda em Semiótica, Tecnologias da Informação e Educação pela UBC, Professora de Clínica Médica da UNICSUL, São Paulo-SP, Brasil.

3. Fisioterapeuta, Mestre em Ciências do Movimento pela UNG, Especialista em Estudos Avançados em Fisioterapia pela UMC, Professora de Fisioterapia Neurofuncional da UNICSUL, São Paulo-SP, Brasil.

4. Fisioterapeuta, pós graduando em Fisioterapia do Trabalho, CBES, São Paulo-SP, Brasil.

5. Fisioterapeuta, Mestre em Reabilitação e Especialista em Gerontologia pela UNIFESP, Professor de Fisioterapia em Saúde Coletiva da UNICSUL, São Paulo-SP, Brasil.
}

Endereço para correspondência: Edna de Souza Cruz Uematsu Clínica de Fisioterapia - Universidade Cruzeiro do Sul Rua Taiuvinha, 31 - Vila Jacuí CEP 08060-040, São Paulo-SP, Brasil Tel.: (11) 2037-5800 E-mail: edna.uematsu@cruzeirodosul.edu.br 


\section{INTRODUÇÃO}

As doenças cerebrovasculares representam as principais causas de morte no Brasil. Dentre essas doenças, o Acidente Vascular Cerebral (AVC) é considerado a causa mais comum de deficiência em adultos, pois quando não leva à morte, normalmente gera incapacidade e dependência ${ }^{1}$. A presença de deficiências motoras são caracterizadas pela fraqueza muscular, alteração do tônus e movimentos estereotipados, que podem limitar as habilidades para realizar atividades como deambular, subir escadas e autocuidar-se ${ }^{2}$.

A incidência do AVC aumenta de modo significativo com a idade, sendo que, dois terços dos acometidos são pessoas acima dos 65 anos. Estima-se que em 2020, quatorze por cento da população brasileira esteja com essa idade e o risco de adquirir a doença dobra a cada 10 anos. Os índices de sobrevivência são altos, sendo que nos indivíduos com idade entre 75 e 84 anos a taxa de sobrevivência é de $79 \%$, acima dos 85 anos essa taxa é de $67 \%{ }^{3}$.

O sinal clínico mais comum é a hemiplegia, que em sua forma mais atenuada, caracterizada pela fraqueza muscular, é denominada hemiparesia, geralmente acompanhada de espasticidade com predomínio na musculatura flexora de membros superiores e extensora de membros inferiores $^{2}$. A fraqueza muscular tem sido reconhecida como fator limitante para pacientes pós-AVC. Até pouco tempo atrás acreditava-se que o fortalecimento muscular poderia exacerbar a restrição imposta pelo músculo espástico e reforçar os padróes anormais de movimento, entretanto, trabalhos que empregaram programas de fortalecimento muscular e/ou condicionamento físico em pacientes hemiparéticos obtiveram ganhos funcionais sem alterar o tônus muscular ${ }^{3,4}$. Por não gerar força em níveis normais, ocorre um aumento no gasto energético para determinada atividade e, consequentemente, os músculos tornam-se mais fadigáveis, levando a um déficit na resistência ${ }^{1,2,5}$. O gasto energético necessário para realizar a deambulação de rotina é elevado em aproximadamente 1,5 a 2 vezes nos indivíduos hemiparéticos comparados com indivíduos saudáveis ${ }^{6}$.

Em relação aos tratamentos, a fisioterapia convencional tem como principais objetivos ganhos motores $\mathrm{e}$ funcionais, como aumento de força muscular e amplitude de movimento, treino de marcha, equilíbrio e coorde- nação, entre outros. Essa abordagem clássica muitas vezes se torna limitada, uma vez que, o paciente pós-AVC possui baixa tolerância ao exercício físico devido à redução da capacidade aeróbica, tendo por consequência um aumento do gasto energético durante a realização de qualquer atividade e uma redução na capacidade de gerar força. Contudo, pacientes hemiparéticos crônicos, quando submetidos a programas de fortalecimento muscular e condicionamento físico, apresentam aumento da capacidade aeróbica, melhora funcional e na qualidade de vida ${ }^{1,4,7-10}$.

Ao longo de semanas de prática regular e repetitiva ao exercício, desenvolvem-se adaptaçôes morfofuncionais, chamadas de efeitos crônicos, que aumentam a capacidade do organismo de responder aos estímulos agudos do exercício. A prática regular de exercícios de resistência evoca uma grande variedade de mudanças fisiológicas que melhoram a habilidade em responder as demandas de exercícios subsequentes. Essas adaptações permitem realizar uma determinada quantidade de trabalho com menos estresse fisiológico, permitindo que se torne mais ativo nas rotinas diárias e possibilitando maior independência ${ }^{11,12}$.

A diminuição do estresse fisiológico proveniente da prática de atividade física possui relação com o aumento do volume máximo de oxigênio consumido (VO${ }_{2}$ máx), que em pacientes hemiparéticos é cerca de 50\% menor quando comparado à população normal ${ }^{13}$. Muitos estudos indicam que pessoas sedentárias dentro de diversas populaçôes experimentam melhoras de $15 \%$ ou mais no VO2máx com apenas três meses de programa de treinamento de resistência, desenvolvendo tolerância para a realização de atividades de vida diária ${ }^{4,6,11}$. Além disso, o trabalho submáximo tende a diminuir a frequência cardíaca (FC) e a pressão arterial (PA) em repouso, melhorando o perfil de risco cardiovascular em indivíduos após o $\mathrm{AVC}^{3,12}$.

Nosso objetivo foi avaliar os efeitos do treinamento em esteira e fortalecimento muscular sobre os sistemas cardiovascular e respiratório, e a mobilidade de indivíduos hemiparéticos crônicos.

\section{MÉTODO}

Este é um estudo quasi-experimental analítico, desenvolvido na Clínica Escola de Fisioterapia da Uni- 
versidade Cruzeiro do Sul, em São Paulo, no período de janeiro a junho de 2009 e aprovado pelo Comitê de Ética para Análise de Pesquisa da Universidade, sob o protocolo no $151 / 2008$.

\section{Amostra}

Participaram deste estudo dez indivíduos de ambos os sexos, com idade superior a 40 anos, hemiparéticos por sequela de AVC isquêmico ou hemorrágico. Como critérios de inclusão foram selecionados para pesquisa indivíduos com tempo de evolução do AVC maior que 6 meses, capazes de realizar exercícios por 45 minutos com intervalos de repouso, deambular por no mínimo 15 minutos com ou sem dispositivos de auxílio para marcha, com exceção de andador e apresentar boa compreensão para seguir as propostas do estudo. Foi solicitado que todos portassem atestado de médico cardiologista liberando-os para atividade física, que assinassem o Termo de Consentimento Livre e Esclarecido, e que estivessem disponíveis por 18 semanas consecutivas durante aplicação do programa de treinamento e testes específicos, além de não estarem realizando tratamento de fisioterapia ou outra atividade de condicionamento. Foram excluídos da pesquisa indivíduos que apresentassem inviabilidade médica atestada por qualquer motivo de saúde que restringisse a participação no programa de condicionamento físico.

\section{Procedimentos de Avaliaçáo}

A avaliação do tratamento aconteceu por meio da comparação de testes específicos de esforço, mobilidade, equilíbrio, pressóes respiratórias máximas e capacidade inspiratória máxima, obtidas antes, durante e após o programa de condicionamento, além da monitorização diária da $\mathrm{PA}, \mathrm{FC}$ e frequência respiratória.

O Teste de Esforço em Esteira foi utilizado por meio do Protocolo de Naughton que avalia as respostas clínicas, hemodinâmicas, eletrocardiográficas e metabólicas ao esforço de indivíduos com limitação física importante. É um exame de baixo custo, de fácil execução e alta reprodutibilidade, aplicado para avaliar a tolerância e causas de limitação dos pacientes ao exercício, delinear uma prescrição segura e adequada para o programa de condicionamento físico, além de avaliar as mudanças ocorridas no pré e pós-treinamento ${ }^{14}$.
Para avaliação da mobilidade foi utilizado o teste POMA que inclui itens de equilíbrio estático e dinâmico, dividido nos subgrupos equilíbrio e marcha. Os testes de equilíbrio são: equilíbrio sentado, levantar e sentar em uma cadeira, equilíbrio em pé (olhos abertos e olhos fechados, suportando uma perturbação, apoio sobre uma perna, apoio com pés alinhados, estendendo os braços à frente, curvando-se para frente) e girar $360^{\circ}$. Os testes de marcha incluem uma avaliação do início da marcha, desvio do trajeto, subida de degraus, giro e tempo de marcha. Os itens são pontuados em uma escala de $0 \mathrm{a} 2 \mathrm{e}$ recebem uma pontuação total de 28 pontos ${ }^{15}$.

A mensuração da pressão respiratória máxima (PRM) foi realizada com um manovacuômetro digital da marca MVD 300, escalonado de 1 em $1 \mathrm{cmH}_{2} \mathrm{O}$, com variaçóes de $-300 /+300 \mathrm{cmH}_{2} \mathrm{O}$. Através da PRM pode-se determinar a gravidade, as consequências e o progresso de diversas disfunçóes pulmonares e neuromusculares. $\mathrm{Na}$ avaliação da pressão inspiratória máxima (PImáx) os participantes foram orientados a realizar uma inspiração forçada a partir do volume residual. Para mensurar a pressão expiratória máxima (PEmáx) os participantes realizaram expiração máxima a partir da capacidade pulmonar total. O exame foi realizado com os participantes sentados, eretos e com os pés apoiados ${ }^{16}$.

Para avaliação da capacidade inspiratória foi utilizado um equipamento Voldyne 5000, em que os participantes foram orientados a inspirar profundamente através do bocal do equipamento até capacidade pulmonar total, partindo da capacidade residual funcional.

\section{Protocolo de treinamento}

As intervençóes foram realizadas 3 vezes por semana, em dias alternados com tempo médio de 80 minutos por sessão durante um período de 4 meses. O protocolo de treinamento incluiu exercícios preparatórios de alongamento muscular passivo e auto-alongamento para membros superiores, inferiores e tronco, com duração média de 10 minutos. Caminhada em esteira ergométrica, seguindo parâmetros individualizados, com tempo de 10 a 35 minutos e velocidade de acordo com a condição de mobilidade do participante. No período inicial foram realizados exercícios de baixa intensidade e de menor tempo, aumentando progressivamente, de acordo 
com o tolerado pelo participante, baseado nos resultados do Teste de Esforço e no acompanhamento de médico cardiologista. Após a esteira, foram realizados exercícios ativos livres e resistidos com uso de halteres, faixas elásticas e bola terapêutica com tempo médio de 15 minutos, seguidos de resfriamento com exercícios respiratórios e de relaxamento global por cerca de 5 minutos.

\section{Análise estatística}

Para análise estatística foi utilizado o Teste $\mathrm{T}$ de Student (pareado) para os dois momentos avaliados do TE. O teste ANOVA (fator duplo sem repetição) foi aplicado em todas as outras variáveis com três momentos avaliados. Para ambos os testes foram utilizados $\alpha$ com nível de significância igual a 0,05.

\section{RESULTADOS}

\section{Caracterizaçáo da Amostra}

A amostra foi constituída de 10 pacientes hemiparéticos por sequela de AVC, sendo 4 homens e $6 \mathrm{mu}$ lheres, com idade variando entre 40 e 74 anos $(54,8 \pm 10)$. Apresentavam tempo de evolução após o primeiro episódio de AVC variando entre 1 e 7 anos ( $5 \pm 2$ anos), $60 \%$ com comprometimento motor de domínio direito e $40 \%$ no esquerdo. Dos 10 participantes apenas 1 era canhoto e $70 \%$ deles tiveram o hemicorpo dominante atingido pela paresia. Os diagnósticos clínicos em 60\% dos casos foram de AVC isquêmico e $40 \%$ hemorrágico. Metade dos participantes utilizava dispositivos de auxílio à marcha (bengala ou muleta canadense) e 40\% órtese de tornozelo/pé. Todos possuíam Hipertensão Arterial Sistêmica como doença associada e faziam uso de mais de um medicamento anti-hipertensivo, sendo estes de mecanismos e classes diferentes. Dentre os fármacos utilizados, 50\% usavam betabloqueadores, $90 \%$ inibidores da enzima de conversão da angiotensina (captopril), 60\% diuréticos tiazídicos (hidroclortiazida) e 20\% bloqueadores dos canais de cálcio (Nifedipina). Todos os pacientes com AVC isquêmicos utilizavam, também, antiagregante.

\section{Teste Ergométrico de Esforço em Esteira (TE)}

$\mathrm{O}$ TE é considerado eficaz quando se atinge, pelo menos, a FC submáxima, que representa $85 \%$ da FC máxima do indivíduo. O teste foi eficaz para $20 \%$ dos participantes na avaliação inicial e ao final do programa para $70 \%$. Na comparaçấo entre os dois períodos avaliados, conforme observado na Tabela 1, houve um aumento significante de 387,6 metros $(145,4 \%)$ na média da distância percorrida pelo grupo. Todos os participantes apresentaram melhor desempenho durante a última avaliação.

$\mathrm{Na}$ avaliação inicial 5 participantes $(50 \%)$ realizaram apenas o primeiro estágio do teste, sendo que destes,

Tabela 1

Resultados obtidos durante aplicação do Teste Ergométrico de Esforço em Esteira no inicio e ao final do programa de treinamento em esteira com pacientes hemiparéticos

\begin{tabular}{lccc}
\hline \multicolumn{1}{c}{ Domínio } & Inicial & Após 4 meses & Valor de $\mathbf{p}$ \\
\hline Estágio Atingido do Teste & $2,5 \pm 2,22$ & $5 \pm 2,35$ & 0,003 \\
\hline Distância Percorrida (metros) & $266,49 \pm 348,83$ & $653,95 \pm 354,99$ & 0,003 \\
\hline $\mathrm{VO}_{2}$ máx $(\mathrm{ml} / \mathrm{kg} / \mathrm{min})$ & $8,6 \pm 7,5$ & $17,8 \pm 9,1$ & 0,002 \\
\hline PA Sistólica Repouso $(\mathrm{mmHg})$ & $130 \pm 7,9$ & $125 \pm 10$ & 0,27 \\
PA Diastólica Pepouso $(\mathrm{mmHg})$ & $90 \pm 5,2$ & $90 \pm 7$ & 0,75 \\
PA Média Repouso $(\mathrm{mmHg})$ & $86,7 \pm 4,5$ & $87,8 \pm 5,3$ & 0,75 \\
FC Repouso (bpm) & $81,5 \pm 10,8$ & $80,5 \pm 10,9$ & 0,75 \\
PA Sistólica Máxima $(\mathrm{mmHg})$ & $171 \pm 20,2$ & $196 \pm 15,8$ & 0,003 \\
\hline PA Diastólica Máxima $(\mathrm{mmHg})$ & $92 \pm 9,2$ & $100 \pm 8,2$ & 0,052 \\
\hline PA Média Máxima $(\mathrm{mmHg})$ & $118,3 \pm 11,2$ & $132 \pm 9,6$ & 0,004 \\
\hline FC Máxima (bpm) & $122,6 \pm 27,9$ & $147,2 \pm 24,9$ & 0,045 \\
\hline
\end{tabular}

Legenda: $\mathrm{PA}=$ Pressão Arterial; $\mathrm{FC}=$ Frequencia Cardíaca; $\mathrm{VO}_{2}$ máx= Pico do Consumo Máximo de Oxigênio 
2 (40\%) não conseguiram atingir a metade do estágio, por apresentarem deficiência acentuada na mobilidade e no equilíbrio. Na segunda avaliação somente uma participante não conseguiu passar para o segundo estágio do teste, porém a dificuldade da participante em realizar a marcha foi muito menor do que na primeira avaliação, conseguindo realizar normalmente o teste enquanto a esteira se manteve na velocidade de $1 \mathrm{mph}$.

Ainda na Tabela 1 é possível observar que a FC e a PA em repouso nos dois momentos avaliados do TE não apresentaram diferenças significativas, porém ao esforço comparando-se os dois momentos houve aumento significante de 20\% na FC Máxima e 11,6\% na PA Média Máxima, devido ao nível de esforço mais alto que os participantes atingiram no teste ao final do programa.

\section{Avaliaçáo Orientada para o Desempenho (POMA I)}

Conforme apresentado no Gráfico 1, houve aumento de $21,4 \%$ na pontuação média geral do grupo. Todos os participantes melhoraram a pontuação total do teste, sendo que 3 participantes (30\%) atingiram a pontuação máxima ao final do programa. O equilíbrio obteve aumento de $24,7 \%$. Neste domínio 9 participantes (90\%) melhoraram a pontuação do teste, sendo que destes, 4 (44,4\%) obtiveram pontuação máxima e 1 (11,1\%) manteve a pontuação da avaliação inicial. A marcha obteve aumento de $14,3 \%$. Neste domínio 8 participantes (80\%) melhoraram a pontuação do teste, sendo que destes, $7(87,5 \%)$ obtiveram pontuação máxima. Os outros 2 (20\%) mantiveram a mesma pontuação da avaliação inicial.

\section{Pressóes e Volumes Respiratórios}

O Gráfico 2 demonstra melhora significante de $55,25 \%$ da PEmáx, com aumento de 20,5 $\pm 25,8 \mathrm{cmH}_{2} \mathrm{O}$ na média entre os participantes do estudo. Com relação à avaliação inicial, 90\% dos participantes apresentaram aumento e $10 \%$ apresentaram redução na PEmáx aferida. Já na PImáx a melhora de 32,6\% observada na média entre os participantes do estudo não foi significante $(\mathrm{p}=0,068)$. Com relação à avaliação inicial $80 \%$ dos participantes apresentaram aumento e $20 \%$ redução na PImáx aferida.

Em relação ao sexo os valores de força muscular respiratória obtidos pelas mulheres foram inferiores aos valores alcançados pelos homens. Houve aumento na PImáx de $43,5 \%$ para as mulheres e de $47 \%$ para os homens, já os valores obtidos na PEmáx aumentaram em $55,3 \%$ e $55,4 \%$ respectivamente.

Como apresentado no Gráfico 3, houve melhora significante $(p<0,001)$ de $57,3 \%$ na capacidade inspiratória máxima com aumento de $860 \mathrm{ml}$ na média entre os participantes do estudo. Com relação à avaliação inicial $80 \%$ dos participantes apresentaram aumento e $20 \%$ não apresentaram mudanças na capacidade inspiratória máxima aferida.

\section{DISCUSSÃO}

A perda da habilidade de andar representa um dos maiores problemas encontrados em pacientes com comprometimentos neuromusculares. Dentre outros fatores, a redução da velocidade da marcha dos indivíduos hemiparéticos está relacionada à espasticidade, alteraçóes de equilíbrio, fraqueza muscular e descondicionamento

físico resultante de inatividade. Existe, ainda, a dificuldade em manter o peso sobre o hemicorpo afetado, o que interfere no controle postural, gerando dificuldades em realizar movimentos de tronco e membros. A idade representa outro fator determinante para o desempenho da mobilidade funcional destes pacientes, quanto mais avançada for, menos eficaz torna-se a marcha em relação ao conforto e velocidade, ocasionando um maior gasto energético ${ }^{8,17}$.
Gráfico 1. Representação dos resultados obtidos durante aplicação do Performance-Oriented Mobility Assessment nos três momentos avaliados do programa de treinamento com pacientes hemiparéticos. 


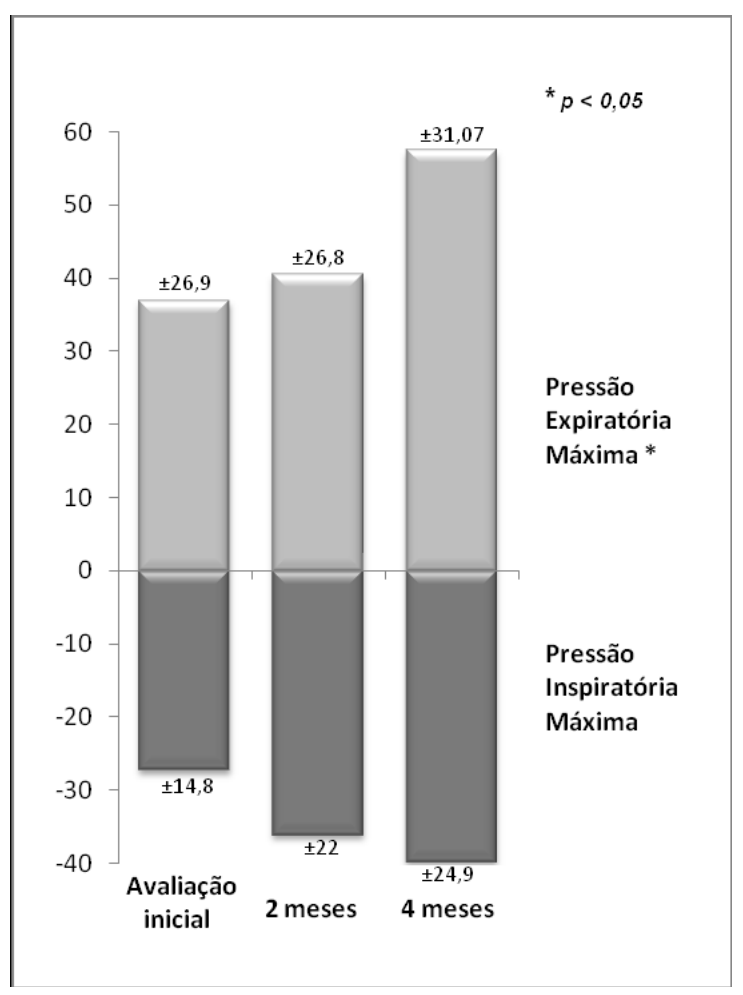

Gráfico 2. Comparação da média obtida nas avaliações das pressões inspiratória e expiratória máxima do grupo de participantes nos três momentos avaliados do programa de treinamento com pacientes hemiparéticos.

O gasto energético durante a marcha em pacientes hemiparéticos varia de acordo com o grau de fraqueza, espasticidade, treinamento e uso de órteses, porém o consumo de oxigênio na caminhada é mais elevado nessa população quando comparados com indivíduos fisicamente saudáveis. O comprometimento motor do AVC faz com que o gasto energético durante a caminhada seja até duas vezes maior, assim como o aumento do gasto energético na execução de AVDs básicas, podendo criar um círculo vicioso de diminuição da atividade e da intolerância ainda maior aos exercícios, levando a complicações secundárias, como a aptidão cardiorrespiratória reduzida, atrofia muscular, osteoporose e insuficiência de circulação nas extremidades inferiores ${ }^{4}$.

Os modelos de reabilitação atuais mais comumente utilizados não são considerados suficientes para a recuperação motora e cardiorrespiratória, pois não proporcionam exercícios adequados para reverter o descondicionamento físico, não possuem tarefas de repetição o suficiente para otimizar a reaprendizagem motora para deambulação e nem a manutenção para que os benefícios sejam mantidos em longo prazo. $\mathrm{O}$ treinamento em esteira pode ser utilizado com intuito de aperfeiçoar a reabilitação motora enquanto condiciona o sistema cardiovascular ${ }^{10}$.

O alto volume de repetiçóes que é necessário para que o paciente realize a marcha na esteira em um padrão mais desejável de simetria e de ativaçáo sensório-motora proporcionam uma exposição a estímulos biomecânicos e neurofisiológicos que promovem adaptaçôes duradouras no desempenho motor, representando um quesito fundamental de aprendizagem de habilidades que é a prática através da repetição automática. No presente estudo foi observado que estas adaptaçóes promoveram mudanças na marcha dos participantes, acarretando melhoras verificadas no decorrer de cada sessão, assim como no Teste de Esforço em Esteira e no POMA, que também verificou a melhora do equilíbrio, fator importante para estabilidade, segurança e prevenção de quedas durante a marcha.

O exercício físico após o AVC é de fundamental importância para o combate e a prevenção ao descondicionamento cardiovascular e das doenças cardiovasculares que acompanham a inatividade física $^{18}$.

A literatura descreve a HAS como o principal fator de risco para AVC, sendo grande a incidência de HAS como doença associada em indivíduos hemiparéticos, casuística observada em todos os participantes deste estudo. É comum encontrá-la presente 
em pacientes com menos de 50 anos vítimas de $\mathrm{AVC}^{19}$, embora um dos principais fatores de risco seja a idade avançada, o que justifica a maioria dos pacientes terem mais de 60 anos. Vários estudos têm verificado a diminuição da idade de indivíduos ao sofrerem AVC de 60 a 74 anos em média para menos de 55 anos, assim como observado nos participantes deste estudo ${ }^{19,20}$.

Pode-se considerar o aumento da prática de exercícios aeróbicos uma estratégia eficaz para a prevenção e tratamento de HAS, visto que na literatura há evidências de que a atividade física de longo prazo reduz os níveis pressóricos de indivíduos normotensos e hipertensos em repouso e durante o exercício ${ }^{21}$. Existe ainda a possibilidade do paciente hipertenso diminuir a dosagem dos seus medicamentos anti-hipertensivos ou até ter a sua PA controlada, como mostram diversos trabalhos que estudaram o efeito do exercício físico sobre a PA, os quais apontaram benefícios, principalmente, sobre os níveis de repouso da $\mathrm{PA}^{22}$.

Durante as verificaçóes de controle da PA, realizadas rotineiramente em três momentos de cada sessão, foram observadas melhoras com redução da PA de repouso e estabilização durante as atividades de esforço. Essas melhoras possibilitaram a redução na medicação anti-hipertensiva de participantes do grupo durante o acompanhamento médico. Assim como sugerido em um estudo com treinamento aeróbico em bicicleta ergométrica, três vezes por semana durante 10 semanas com indivíduos hemiparéticos crônicos, em que os autores observaram diminuição dos valores pressóricos, que poderiam servir de complemento para se atenuar a dosagem das drogas anti-hipertensivas ${ }^{23}$. Em outro estudo com seis meses de atividade física regular, foi observado que a PA, que no início era alta e instável, após treinamento aeróbico de longa duração e baixa intensidade, manteve-se estável e dentro dos limites considerados normais ${ }^{24}$.

O treinamento aeróbico tende a reduzir, também, a FC em repouso e durante o exercício realizado em cargas submáximas de trabalho. Esses efeitos podem ser atribuídos à redução da hiperatividade simpática e aumento da atividade parassimpática, mudança no marca-passo cardíaco ou mesmo na melhora da função sistólica. A FC máxima não é alterada de modo apreciado, porém após condicionamento serão necessários níveis mais intensos de esforço para que essa FC máxima seja alcançada ${ }^{25}$. Neste estudo náo foram observadas diferenças na FC de repouso após o programa de tratamento, contudo a frequência manteve-se nos padróes de normalidade durante todo o programa. Todos os participantes faziam uso da medicação anti-hipertensiva, mesmo aqueles em uso de betabloqueador, o que, por precaução, limitou a FC máxima na realização do TE, para evitar uma possível crise hipertensiva.

Em um estudo realizado com pacientes hemiparéticos, após programa de condicionamento aeróbico, não foram observadas alteraçóes na FC com o teste de caminhada de seis minutos após o programa, contudo a velocidade da marcha obteve um aumento significante, assim como a distância percorrida no teste, exigindo uma FC que suprisse as condiçóes hemodinâmicas ${ }^{8}$.

A atividade física é um fator relevante para o envelhecimento saudável e parece haver, também, correlação entre atividade física geral e o aumento da força dos músculos respiratórios, embora não sejam exercícios direcionados a esta finalidade. Os valores quanto à força muscular obtidos no presente estudo ao final do programa de treinamento mantiveram-se abaixo do esperado para indivíduos saudáveis da mesma idade e sexo ${ }^{16}$. Para os homens, a PEmáx obtida foi $70 \%$ da estimada e a PImáx $60 \%$, enquanto para as mulheres, a PEmáx obtida foi $35,6 \%$ da estimada e a PImáx 40\%. No entanto, observou-se melhora significante da força muscular expiratória e melhora clinicamente significativa da força muscular inspiratória nestes participantes, com aumento também significante da capacidade inspiratória máxima.

A diferença nos ganhos de PImáx e PEmáx pode ser explicada pelo fato que, durante a respiração normal, a inspiração se dá por um processo mecânico ativo, o que exige contração muscular, mesmo que diminuída, fazendo com que a musculatura inspiratória mantenha um mínimo de força e condicionamento. Já a expiração em situações normais é um processo passivo, sem a necessidade de contração muscular. Ao submeter um indivíduo a um programa de condicionamento aeróbico, exige-se dele um trabalho de força e resistência muscular, tanto de músculos inspiratórios, como de músculos expiratórias. Estes últimos por permanecerem inativos a maior parte do tempo, após o programa de condicionamento, evi- 
denciam melhora mais significante que os inspiratórios que já se encontravam em melhores condiçóes, sofrendo alteraçóes em proporçóes menores para atingir o ideal. $\mathrm{O}$ mesmo resultado foi observado em um estudo realizado por meio de treinamento aeróbico em indivíduos normais, em que não houve diferença antes do treinamento muscular na PImáx e PEmáx, porém, após o treinamento a PEmáx aumentou significativamente em 25,4\%. Enquanto que a PImáx, apesar de melhorar, não apresentou alterações significantes ${ }^{26}$.

A espasticidade em pacientes hemiparéticos, quando acentuada no abdômen dificulta a ação diafragmática, levando a um desconforto respiratório e ao uso da musculatura acessória ${ }^{27}$. Além disso, a PImáx e PEmáx apresentam valores inferiores ao previsto para a idade, indicando a diminuição da força dos músculos respiratórios nesses indivíduos ${ }^{5}$. Tendo em vista que os pacientes hemiparéticos apresentam complicaçóes cardiorrespiratórias devido à imobilidade, a prática de atividade física, por meio de exercícios aeróbicos, pode ser considerada como forma de amenizar este quadro. É necessário que a atividade física seja mantida após o condicionamento, de outro modo ocorrerá perda gradativa da força muscular respiratória adquirida $^{27}$.

Foi observado melhora significante na distância percorrida e no pico do consumo máximo de oxigênio $\left(\mathrm{VO}_{2}\right.$ máx $)$ dos participantes deste estudo durante o TE, o que vai ao encontro com outros estudos que ao utilizarem protocolos de condicionamento aeróbico verificaram aumento no $\mathrm{VO}_{2}$ máx e diminuição do gasto energéti$\mathrm{Co}^{10,28}$. O VO${ }_{2}$ máx avalia de forma específica a capacidade aeróbica de um indivíduo. $\mathrm{O}$ sistema de transporte do oxigênio sofre uma adaptação favorável com o treinamento físico, que se exterioriza através de maiores valores de $\mathrm{VO}_{2}$ máx. $\mathrm{O}$ treinamento físico aumenta a diferença arteriovenosa de oxigênio através do aumento da volemia, da densidade capilar, do débito cardíaco e da extração periférica de oxigênio durante o exercício ${ }^{25}$. Em geral, a absorção de oxigênio em uma determinada carga de trabalho submáxima em pacientes que sofreram AVC é maior do que em indivíduos saudáveis, possivelmente devido à reduzida eficiência mecânica, os efeitos da espasticidade, ou ambos, apresentando um elevado consumo de oxigênio, principalmente durante atividades como a marcha.
Em contraste, $\mathrm{o} \mathrm{VO}_{2}$ máx é reduzido nesses indivíduos. Aqueles que estão mais aptos possuem maiores valores de $\mathrm{VO}_{2}$ máx podendo exercer atividades físicas mais inten$\operatorname{sas}^{4,29,30}$.

O elevado gasto energético nos indivíduos com AVC implica em importantes perdas e interfere diretamente na qualidade de vida dos mesmos. Esta diminuição da capacidade funcional causada pelo alto custo de oxigênio gera dificuldades na execução das atividades básicas e instrumentais de vida diária. A reduzida aptidão física pode ser um fator secundário, que limita a utilização das respostas adquiridas durante o processo de reabilitação e consequentemente o potencial para restabelecer sua independência física ${ }^{26}$. O gasto energético para realizar as atividades de vida diária pode ser menor se houver uma melhora da capacidade cardiovascular através de um treinamento aeróbico, o que permite que estas atividades sejam efetuadas com um menor percentual energético ${ }^{3,4}$.

O exercício aeróbico em longo prazo tende a proporcionar condicionamento físico, no qual, ao se realizar uma determinada tarefa de vida diária ou atividade, será exigido uma menor proporção de volume de oxigênio suficiente para suprir o gasto energético, além de poderem realizar atividades por um tempo prolongado, com menos fadiga. Este benefício previne ou retarda os prejuízos funcionais após o $\mathrm{AVC}^{26,31}$. Outro fator a ser considerado na inclusão desta modalidade de tratamento, é que o aumento da capacidade aeróbica relaciona-se com a melhora global da função sensório-motora, visto que, o treinamento aeróbico proporciona adaptaçóes neurológicas que aumentam o recrutamento de unidades motoras, promovendo uma adaptação sinérgica entre os grupos musculares, que melhora a coordenação neuromuscular e reduz a energia requerida para realização de atividades ${ }^{3,11,12}$.

O pequeno tamanho amostral do presente estudo, de certa forma, pôde ser compensado pela homogeneidade dos resultados obtidos durante os testes, assim como a compatibilidade com a literatura existente que demonstra a eficiência da fisioterapia através dos programas de treinamento em esteira e fortalecimento muscular no tratamento de pacientes hemiparéticos em decorrência de AVC. Outras limitaçóes do estudo foram a ausência de avaliaçôes com métodos mais complexos e dispendiosos como diluição de gases inertes, ausência dos níveis 
de saturação, níveis de lactato durante o TE, análise da atividade autonômica do sistema cardiovascular e análise biomecânica detalhada durante a marcha.

Novos estudos devem ser realizados, principalmente no que se refere à qualidade de vida destes pacientes, tendo como principais objetivos o reforço aos resultados já encontrados e o incentivo a introdução destes recursos nos programas de fisioterapia mais comumente utilizados, sobretudo nos programas de saúde pública, visto que este é um método de intervenção relativamente barato, que apesar da atenção individualizada para cada paciente, pode ser aplicado de forma coletiva. Essas intervençóes além de tudo poderiam contribuir no desafogamento dos serviços de saúde, na diminuição da quantidade de medicamentos administrados a essa população, e num possível retorno desses indivíduos a uma vida social e economicamente ativa.

\section{CONCLUSÃO}

Os resultados encontrados neste estudo sugerem que o treinamento em esteira associado ao fortalecimento muscular pode melhorar a capacidade funcional, influenciada pelo desenvolvimento da mobilidade e do equilíbrio, e, consequentemente, produzir adaptaçóes cardiorrespiratórias, como a diminuição da hipertensão arterial, aumento do consumo máximo de oxigênio, da força muscular respiratória e da capacidade inspiratória máxima, favorecendo ao menor gasto energético, possibilitando melhor desempenho na execução de atividades físicas.

\section{AGRADECIMENTOS}

Ao Mello Centro de Diagnósticos e a coordenação do curso de Fisioterapia da Universidade Cruzeiro do Sul.

\section{REFERÊNCIAS}

1.Teixeira-Salmela LF, Lima RCM, Lima LAO, Morais SG, Goulart F. Assimetria e desempenho funcional em hemiplégicos crônicos antes e após programa de treinamento em academia. Rev Bras Fisioter 2005;9:227-33.

2.Oliveira RJ. Atividade física e doença cerebrovascular. Rev Bras Ciênc Mov 2001;9:70-8.

3.Teixeira-Salmela LF, Oliveira ESG, Santana EGSS, Resende GP. Fortaleci- mento muscular e condicionamento físico em hemiplégicos. Acta Fisiátrica 2000;7:108-18.

4.Gordon NF, Cochair MG, Gulanick M, Costa F, Fletcher G, Franklin BA, et al. Physical activity and exercise recommendations for stroke survivors. Stroke 2004;35:1230-40.

http://dx.doi.org/10.1161/01.STR.0000127303.19261.19

5.Fernandes MA, Freitas BHPF, Negrini F, Sampaio LMM, Medalha CC. Contribuiçôes da avaliação cardiorrespiratória em pacientes hemiplégicos. Arq Sanny Pesq Saúde 2008;1:90-7.

6.Luft A, Macko RF, Forrester LW, Goldberg A, Hanley DF. Post-stroke exercise rehabilitation: What we know about retraining the motor system and how it may apply to retraining the heart. Cleveland Clinic Journal of Medicine 2008;75:83-6.

http://dx.doi.org/10.3949/ccjm.75.Suppl_2.S83

7.Teixeira-Salmela LF, Faria CDCM, Guimarães CQ, Goulart F, Parreira VF, Inácio EP, et al. Treinamento físico e destreinamento em hemiplégicos crônicos: impacto na qualidade de vida. Rev bras fisioter 2005;9:347-53.

8. Teixeira-Salmela LF, Silva PC, Lima RCM, Augusto ACC, Souza AC, Goulart F. Musculação e condicionamento aeróbio na performance funcional de hemiplégicos crônicos. Acta Fisiátrica 2003;10:54-60.

9.Rimmer JH, Wang E. Aerobic exercise training in stroke survivors. Top Stroke Rehabil 2005;12:17-30.

http://dx.doi.org/10.1310/L6HG-8X8N-QC9Q-HHM8

10.Macko RF, Ivey FM, Forrester LW, Hanley D, Sorkin JD, Katzel LI, et al. Treadmill exercise rehabilitation improves ambulatory function and cardiovascular fitness in patients with chronic stroke: a randomized, controlled trial. Stroke 2005;36:2206-11.

http://dx.doi.org/10.1161/01.STR.0000181076.91805.89

11.Frontera WR, Dawson DM, Slovik DM. Exercício físico e reabilitação. Porto Alegre: Artmed, 2001, p.59-70.

12.Araújo CGS. Fisiologia do exercício físico e hipertensão arterial: uma breve introdução. Rev Hipertens 2001;4:78-83.

13.Michael K, Macko RF. Ambulatory activity intensity profiles, fitness, and fatigue after stroke. Top Stroke Rehabil 2007;14:5-12.

http://dx.doi.org/10.1310/tsr1402-5

14.Mastrocolla LE, Brito AX, Brito FS, Castro I, Godoy M, Alfieri RG, et al. Consenso nacional de ergometria. Arq Bras Cardiol 1995;65:417-23.

15.Tinneti ME. Performance-oriented assessment of mobility in elderly. J Am Geriatr Soc 1986;34:119-26.

16.Neder JÁ, Andreoni S, Lerario MC, Nery LE. Reference values for lung function tests. II. maximal respiratory pressures and voluntary ventilation. Braz J Med Biol Res 1999;32:719-27.

http://dx.doi.org/10.1590/S0100-879X1999000600007

17.Visintin M, Barbeau H, Korner-Bitensky N, Mayo NE. Support and treadmill stimulation a new approach to retrain gait in stroke patients through body weight. Stroke 1998;29:1122-8.

18.Macko RF, Ivey FM, Forrester LW. Task-oriented aerobic exercise in chronic hemiparetic stroke: training protocols and treatment effects. Top Stroke Rehabil 2005;12:45-57.

http://dx.doi.org/10.1310/PJQN-KAN9-TTVY-HYQH

19.Pires SL, Glagliardi RJ, Gorzoni ML. Estudo da frequência dos principais fatores de risco para acidente vascular cerebral isquêmico em idosos. Arq Neuropsiq 2004;62(3B):844-851.

http://dx.doi.org/10.1590/S0004-282X2004000500020

20.Duncan P, Studenski S, Richards L, Steven G, Sue ML, Dean R, et al. Randomized clinical trial of therapeutic exercise in subacute stroke. Stroke 2003;34:2173-80.

http://dx.doi.org/10.1161/01.STR.0000083699.95351.F2 
21.Mediano MFF, Aragão AHBM, Chame F, Barbosa JSO, Batista LA. Efetividade de um programa de exercícios físicos sobre níveis tensionais em hipertensos controlados. Braz J Biom 2008;2:78-88.

22.Monteiro MF, Sobral-Filho DC. Exercício físico e o controle da pressão arterial. Ver Bras Med Esp 2004;10:513-6.

http://dx.doi.org/10.1590/S1517-86922004000600008

23.Potempa K, Lopez M, Braun LT, Szidon JP, Fogg L, Tincknell T. Physicological outcomes of aerobic exercise training in hemiparetic stroke patients. Stroke 1995;26:101-5.

24.Costa AM, Duarte E. Atividade física e a relação com a qualidade de vida de pessoas com sequelas de acidente vascular isquêmico (AVCI). Rev Bras Ciên e Mov 2002;10;47-54.

25.Moraes RS, Nóbrega ACL, Castro RRTC, Negrão CE, Stein R, Serra SM, et al. Diretriz de reabilitação cardíaca. Arq Bras Cardiol 2005;84:431-40.

26.Suzuky S, Sato M, Okubo T. Expiratory muscle training and sensation of respiratory effort during exercise in normal subjects. Thorax 1995;50:366-70. http://dx.doi.org/10.1136/thx.50.4.366
27.Caromano FA, Ide MR, Kerbauy RR. Manutençấo na prática de exercícios por idosos. Rev Dep Psicol UFF 2006;18:177-92.

28.Luft AR, Macko RF, Forrester LW, Villagra F, Ivey F, Sorkin JD, et al. Treadmill exercise activates subcortical neural networks and improves walking after stroke: a randomized controlled trial. Stroke 2008;39:3341-50.

http://dx.doi.org/10.1161/STROKEAHA.108.527531

29.Fredrickson E, Ruff RL, Daly JJ. Physiological cost index as a proxy measure for the oxygen cost of gait in stroke patients. Neuroreh Neural Repair 2007;21:429-34.

http://dx.doi.org/10.1177/1545968307300400

30.Resnick B, Michael K, Shaughnessy M, Nahm EU, Kopunek S, Sorkin J, et al. Inflated perceptions of physical activity after stroke: pairing self-report with physiologic measures. J Phys Act Health 2008;5:308-18.

31.Macko RF, Smith GV, Dobrovolny CL, Sorkin JD, Goldberg AP, Silver $\mathrm{KH}$. Treadmill training improves fitness reserve in chronic stroke patients. Arch Phys Med Rehabil 2001;82:879-84.

http://dx.doi.org/10.1053/apmr.2001.23853 